\title{
Place des troubles de la spermatogenèse dans l'hypofertilité masculine
}

\author{
H. LEJEUNE ${ }^{1,2,3}$, M. BENCHAIB ${ }^{1,3}$, V. BIED ${ }^{1}$, A. BOUCHER $^{2}$, D. BOULIEU $^{4}$, \\ J.C. CZYBA ${ }^{1}$, P. GALLOT-LAVALLEE ${ }^{1}$, J.F. GUERIN ${ }^{1,3}$, S. HADJ ${ }^{1}$, J. LORNAGE ${ }^{1,3}$, \\ I. PLOTTON ${ }^{1,2}$, M. PUGEAT ${ }^{2}$, B. SALLE ${ }^{1,3}$.
}

1 Département de Médecine de la Reproduction, Hospices Civils de Lyon, Hôpital Édouard Herriot, 2 Clinique Endocrinologique, Hospices Civils de Lyon, Hôpital de l'Antiquaille, 3INSERM-INRA U418, Hôpital Debrousse, 4Service de Gynécologie, Hospices Civil de Lyon, Hôpital Edouard Herriot, Lyon

\section{RÉSUMÉ}

Selon l'étude de Thonneau et al. en 1991, 14\% des couples ont des difficultés pour concevoir, dans au moins $59 \%$ des cas, un facteur masculin intervient dans l'infertilité de couple, ce qui permet d'estimer que $8 \%(14 \% \mathrm{x}$ $59 \%$ ) des hommes présentent une hypofécondité. Du fait de la multiplicité des causes d'infertilité masculine et de l'hétérogénéité des critères et classifications utilisées dans la littérature, il est difficile de préciser le pourcentage de chacune des causes d'infertilité. Sur une population de 2072 patients consécutivement vus par le même praticien pour infertilité masculine, on conclut à un trouble de la spermatogenèse dans $52 \%$ des cas ; ce qui suggère qu'environ $4 \%$ des hommes présentent un trouble de la spermatogenèse. Le trouble de la spermatogenèse est isolé dans $43 \%$ des cas (soit $23 \%$ des patients vus en consultation d'infertilité masculine) ou associé à des altérations " post-testiculaires" (infectieuses/inflammatoires, autoimmunes et/ou obstructives) dans 57\% des cas (soit 30\% des patients). Un facteur étiologique est identifié cliniquement pour $64 \%$ des patients présentant un trouble de la spermatogenèse. Les facteurs de risque les plus fortement associés aux troubles de la spermatogenèse sont : d'une part les antécédents d'orchite ourlienne (OR [IC95\%]=14,6 [3,4-62,3]) et de radiothérapies-chimiothérapies $(\mathrm{OR}=14,7[3,4-63,2])$, situations relativement peu fréquentes $(1,4 \%$ et $1,3 \%$ des cas) mais génératrices de troubles de la spermatogenèse dans une proportion élevée de cas $(92,9 \%$ et $92,3 \%)$ et d'autre part les varicocèles $(\mathrm{OR}=3,7 \quad[2,9-4,8])$ et les troubles de la descente testiculaire $(O R=2,9$ [2,3-3,7]), qui sont les facteurs de risques les plus fréquents $(20,6 \%$ et $20,1 \%$ des cas) mais sont moins régulièrement associés à un trouble de la spermatogenèse $(73,7$ et $69,6 \%$ des cas).

Mots clés : infertilité masculine - spermatogenèse physiopathologie - étiologie.

\section{INTRODUCTION}

Selon l'étude épidémiologique réalisée sur trois départements français par Thonneau et al. [10], 14\% des couples ont des difficultés pour concevoir. Dans cette étude, le bilan systématique des deux partenaires permet de retenir un facteur masculin soit isolé, dans $20 \%$ des cas, soit associé à un facteur féminin, dans $39 \%$ des cas ; un facteur féminin isolé, dans $32 \%$ des cas ; $8 \%$ des infertilités restent inexpliquées. Ainsi, dans au moins $59 \%$ des cas, un facteur masculin intervient dans l'infertilité de couple, ce qui permet d'estimer que $8 \%(14 \% \times 59 \%)$ des hommes vivant en couple présentent une hypofécondité. Les causes d'infertilité masculine sont variées, plusieurs études ont tenté d'en dresser une classification 
et de déterminer la proportion relative de chacune d'entre elles. On note de grandes variations dans les proportions relatives des causes d'infertilité masculine selon les publications [7]. Ces variations peuvent être dues à des biais de recrutement selon les équipes mais aussi à des différences dans les classifications et critères diagnostiques utilisés. On note à cet égard que pour beaucoup de facteurs étiologiques, la relation de cause à effet avec l'infertilité reste discutée. D'autre part, dans la plupart des études, les auteurs ont cherché à faire entrer chaque patient dans un cadre étiologique unique en ne retenant pour chaque patient qu'une seule cause d'infertilité.

Notre expérience, basée sur une série de patients étudiés de manière homogène, nous pousse

$1^{\circ}$ ) à considérer la possibilité de causes multiples d'infertilité masculine pour chaque patient,

$2^{\circ}$ ) à utiliser plusieurs niveaux de classification et en particulier une classification physiopathologique intermédiaire entre la classification spermiologique communément utilisée et les classifications étiologiques considérant divers facteurs de risques dont la responsabilité est plus ou moins formellement établie et

$3^{\circ}$ ) à estimer la relation statistique entre la présence d'un trouble de la spermatogenèse et la présence des facteurs étiologiques de troubles de la spermatogenèse.

\section{MATÉRIEL ET MÉTHODES}

\section{Population étudiée}

La population étudiée comporte 2072 patients consécutifs, vus par le même praticien (H.L.), pour le bilan de la participation masculine à une infertilité de couple. Chaque patient a eu un interrogatoire et un examen clinique systématisés dont les résultats ont été informatisés à la suite de la consultation.

\section{a) Classification spermiologique}

Dans cette étude consacrée plus spécifiquement aux troubles de la spermatogenèse, les données spermiologiques sont basées sur la numération des spermatozoïdes selon la classification suivante :
- Anéjaculation : absence d'éjaculation antérograde (anéjaculation et éjaculation rétrograde);

- Azoospermie : absence de spermatozoïdes à l'examen direct du sperme, regroupant les azoospermies complètes avec absence de spermatozoïde dans le culot centrifugation du sperme et les cryptozoospermies avec absence de spermatozoïde à l'examen direct mais présence de quelques spermatozoïdes à l'examen du culot de centrifugation ;

- Oligospermie : numération $<40.106$ spermatozoïdes par éjaculât ;

- Numération normale : numération $\geq$ 40.106/ éjaculât.

\section{b) Classification physiopathologique}

Trois mécanismes physiopathologiques pouvant être en cause dans l'altération du spermogramme ont été considérés [3] : les causes "pré-testiculaires" correspondent à un déficit de stimulation du testicule par les gonadotrophines, les causes "testiculaires" correspondent à une altération de la spermatogenèse elle-même et les causes "post-testiculaires" correspondent à une altération des spermatozoïdes après qu'ils aient été produits dans le tube séminifêre, il peut alors s'agir d'une altération des spermatozoïdes par des phénomènes infectieux, inflammatoires ou auto-immunitaires ou encore d'une obstruction des voies séminales. Ces mécanismes physiopathologiques ont été retenus selon les critères suivants : Causes pré-testiculaires : testostérone et gonadotrophines inférieures à la normale ; Causes testiculaires : troubles de la spermatogenèse constatés histologiquement et/ou élévation de la concentration plasmatique de FSH à plus de 3 déviations standard au-dessus de la moyenne de la population de référence (partenaire de patientes présentant une obstruction tubaire et spermogramme normal et segmentation de plus de $50 \%$ des ovocytes) et/ou volume de l'un des testicules, mesuré à l'aide d'un orchidomètre, inférieur à $15 \mathrm{ml}$, à l'exclusion des patients présentant un déficit gonadotrope (catégorie pré-testiculaire) ; Causes post-testiculaires : altération des voies excrétrices ou des spermatozoïdes pendant leur trajet dans les voies excrétrices 
regroupant :

1) obstructions des voies séminales attestées par des arguments morphologiques (chirurgie, échographie)

2) infections/inflammations génitales attestées par une spermoculture positive et/ou une leucospermie (> 106/ml) et/ou une épididymite et/ou une prostatite constatées cliniquement

3) autoimmunisation antispermatozoïde attestée par un MAR test positif ou la présence d'anticorps antispermatozoïdes dans le liquide séminal.

\section{c) Facteurs étiologiques des troubles de la spermatogenèse}

Les facteurs de risque de trouble de la spermatogenèse considérés sont les suivants : Varicocèle constaté à l'examen clinique (grade 1 à 3) ; Trouble de la descente testiculaire, soit antécédents de cryptorchidie traitée chirurgicalement ou médicalement dans l'enfance, antécédents de hernie inguinale de l'enfant traité chirurgicalement et/ou testicules rétractiles constatés à l'examen clinique ; Hyperthermie testiculaire par exposition professionnelle ou récréative (sauna, bains chauds prolongés fréquents) à des chaleurs externes élevées ou excès pondéral avec adiposité pubienne et testicules en position d'hyperthermie relative; Exposition à des toxiques soit d'origine professionnelle (pesticides, insecticides, solvants, peintures), soit par addiction (opiacés, cannabis, éthylisme), soit médicamenteux (Salazopyrine, Colchicine) ; antécédents de Traumatisme testiculaire suivi de la constitution d'un hématome scrotal ; antécédents de Torsion testiculaire ayant motivé une intervention chirurgicale ou une hospitalisation pour observation ; antécédents d'Orchite ourlienne ; antécédents de ChimioRadiothérapies pour pathologie maligne ; constatation ou antécédent de cure chirurgicale d'Hypospadias.

\section{RÉSULTATS}

Le tableau 1 donne le nombre et la proportion de patients en fonction de la classification basée sur la numération de spermatozoïdes. Le nombre et le pourcentage des patients entrant dans les trois catégories physiopathologiques "pré-testiculaire", "testiculaire" ou "post-testiculaire" sont présentés dans le tableau 2. On note que $14,5 \%$ des patients $(n=300)$ ne peuvent entrer dans aucune des catégories physiopathologiques. Du fait des critères utilisés, les catégories physiopathologiques "pré-testiculaire" et "testiculaire" sont exclusives l'une de l'autre. Par contre, un même patient peut présenter à la fois un déficit gonadotrope (pré-testiculaire) ou un trouble de la spermatogenèse (testiculaire) et une pathologie des voies excrétrices (post-testiculaire). La fréquence de l'association des causes testiculaires ou pré-testiculaires avec une (ou des) cause(s) post-testiculaire(s) est présentée dans la figure 1 . L'association de causes post-testiculaire et testiculaire est numériquement notable : sur un total de 1083 patients présentant un trouble de la spermatogenèse, celui-ci est isolé dans $43,3 \%$ des cas et associé à une pathologie posttesticulaire dans $56,7 \%$ des cas.

Le nombre et le pourcentage des patients présentant les facteurs de risques de trouble de la spermatogenèse sont présentés dans le tableau 3. Les varicocèles et les troubles de la descente testiculaires représentent les facteurs de risque les plus fréquents (présents respectivement chez $20,6 \%$ et $20,1 \%$ des patients). Au total, $51 \%$ des patients présentent un ou plusieurs facteur(s) de risque de trouble de la spermatogenèse, 49\% n'en présentent aucun. La relation entre la présence des facteurs de risques et la présence effective d'un trouble de la spermatogenèse (cause testiculaire) a été étudiée pour chacun des facteurs de risque, individuellement (Tableau 4A) et pour l'ensemble des facteurs de risque dans un modèle de régression logistique (Tableau 4B). Les deux facteurs de risques les plus fréquents (varicocèles et troubles de la descente testiculaire) sont significativement associés à un trouble de la spermatogenèse. Deux autres facteurs de risques, les antécédents d'orchite ourlienne et les antécédents de radiothérapie et/ou chimiothérapie sont moins fréquents dans la population étudiée $(1,4 \%$ et $1,3 \%)$ mais donnent lieu à un trouble de la spermatogenèse dans un pourcentage élevé de cas $(92,9 \%$ et $92,3 \%)$. Dans notre population de patients consultant pour infertilité masculine, les autres facteurs de risque, ne sont pas significativement plus fré- 
Tableau 1. Distribution des patients selon la numération des spermatozoüdes. $n$ : nombre de patients ; \% : pourcentage par rapport au nombre total de patients.

\begin{tabular}{lrr}
\hline & $\mathbf{n}$ & $\%$ \\
Anéjaculation & 22 & $1,1 \%$ \\
Azoospermie & 256 & $12,4 \%$ \\
Oligospermie & 1043 & $59,6 \%$ \\
Numération normale & 751 & $36,2 \%$ \\
Total & 2072 & $100,0 \%$ \\
\hline
\end{tabular}

Tableau 2. Physiopathologie des infertilités masculines : $n=$ nombre de patients relevant de la catégorie physiopathologique considérée; \% = pourcentage des patients relevant de la catégorie physiopathologique, rapporté aux 2072 patients étudiés. Un même patient peut entrer dans plusieurs catégories physiopathologiques (voir figure 1).

\begin{tabular}{lrr}
\hline & $\mathbf{n}$ & $\%$ \\
Causes Pré-testiculaires & 22 & $1,1 \%$ \\
Causes Testiculaires : & 1083 & $52,3 \%$ \\
Causes Post-testiculaires : & 1287 & $62,1 \%$ \\
- Obstructions des voies séminales & 127 & $6,1 \%$ \\
- Infections/inflammations génitales & 1157 & $55,8 \%$ \\
- Autoimmunisations & 215 & $10,4 \%$ \\
Physiopathologie indéterminée & 300 & $14,5 \%$ \\
\hline
\end{tabular}

Tableau 3. Fréquence des facteurs de risque de troubles de la spermatogenèse. $n$ : nombre de patients présentant le facteur de risque considéré. \%: pourcentage des patients présentant le facteur de risque considéré rapporté au nombre total de patients. A : effectif pour chaque facteur de risque, un même patient peut présenter plusieurs facteurs de risque. B. Présence ou non d'un ou plusieurs facteur(s) de risque.

$\mathbf{A}$

Varicocèle

Trouble de la descente testiculaire

Hyperthermie testiculaire

Exposition à des toxiques

Traumatisme testiculaire

Torsion testiculaire

Orchite

Chimio-Radiothérapies

Hypospadias

B

Présentant un/des facteur(s) de risque

PAS de facteur de risque

Total des patients

$\begin{array}{rr}\mathbf{n} & \% \\ 426 & 20,6 \% \\ 418 & 20,1 \% \\ 173 & 8,3 \% \\ 140 & 6,8 \% \\ 88 & 4,2 \% \\ 30 & 1,4 \% \\ 28 & 1,4 \% \\ 26 & 1,3 \% \\ 11 & 0,5 \% \\ \mathbf{n} & \% \\ 1056 & 51,0 \% \\ 1016 & 49,0 \% \\ 2072 & 100,0 \%\end{array}$


Tableau 4. Relation entre les facteurs de risque de troubles de la spermatogenèse et les troubles de la spermatogenèse : Tableau $4 A$ : analyse de chaque facteur de risque individuellement : a \% de patients présentant le facteur de risque considéré rapporté aux 2072 patients étudiés; b \% de patients présentant un trouble de la spermatogenèse parmi les patients présentant le facteur de risque considéré ; c valeur de $p$ du test de $X^{2}$ étudiant la liaison entre le facteur de risque considéré et le trouble de la spermatogenèse; $d \%$ de patients présentant le facteur de risque considéré rapporté aux 1083 patients présentant un trouble de la spermatogenèse. Tableau $4 B$ : analyse multifactorielle par régression logistique. $95 \%$ inf. et $95 \%$ sup. sont les bornes supérieures et inférieures de l'intervalle de confiance à $95 \%$ des Odds Ratio.

$\mathbf{A}$

Varicocèle

Trouble descente testiculaire

Hyperthermie testiculaire

Exposition à des toxiques

Traumatisme testiculaire

Torsion testiculaire

Orchite

Chimio-Radiothérapie

Hypospadias

Facteur(s) de risque

PAS de facteur de risque
$\% / 2072 \%$ de trouble patientsa spermatogb

$20,6 \%$

$20,1 \%$

$8,3 \%$

$6,8 \%$

$4,2 \%$

$1,4 \%$

$1,4 \%$

$1,3 \%$

$0,5 \%$

$51,0 \%$

$49,0 \%$

\begin{abstract}
$73,7 \%$
\end{abstract}
$69,6 \%$

$48,0 \%$

$56,4 \%$

$52,3 \%$

$66,0 \%$

$92,9 \%$

$92,3 \%$

$63,6 \%$

$65,8 \%$

$38,2 \%$
$X^{2} \mathrm{c}$

$\mathbf{p}$

$<0,0001$

$<0,0001$

0,2657

0,3355

0,9999

0,1405

$<0,0001$

$<0,0001$

0,5524

$<0,0001$

$<0,0001$
\% / 1083

Trble Sptgd

$29,0 \%$

$26,9 \%$

$7,7 \%$

$7,3 \%$

$4,2 \%$

$1,8 \%$

$2,4 \%$

$2,2 \%$

$0,6 \%$

$64,2 \%$

$35,8 \%$
B

Varicocèle

Trouble descente testiculaire

Hyperthermie testiculaire

Exposition à des toxiques

Traumatisme testiculaire

Torsion testiculaire

Orchite

Chimio-Radiothérapie

Hypospadias
Odds Ratio

$$
3,74
$$

2,935

0,775

1,292

1,074

1,57

14,566

14,748

1,55
95\% inf.

2,933

2,311

0,556

0,895

0,684

0,707

3,408

3,441

0,432
95\% sup.

4,768

3,728

1,081

1,865

1,686

3,487

62,251

63,211

5,555

\section{p}

$<0,0001$

$<0,0001$

0,1328

0,1714

0,7569

0,2682

0,0003

0,0003

0,5013 
quents chez les sujets présentant un trouble de la spermatogenèse que chez ceux n'en présentant pas. De manière globale, $64,2 \%$ des patients présentant un trouble de la spermatogenèse, présentent un ou plusieurs facteur(s) de risque, alors que pour $35,8 \%$ des patients présentant un trouble de la spermatogenèse, aucun des facteurs de risque étudiés ne peut être identifié. Sur le plan statistique, les patients ayant une infection et/ou inflammation génitale (Odds Ratio [intervalle de confiance $95 \%]=0,767[0,644-0,913]$; $\mathrm{p}=0,0028$ ) et surtout une autoimmunisation anti-spermatozoïde (OR $=0,270$ [0,196-0,372] ; $\mathrm{p}<0,0001$ ) ont un risque diminué d'avoir un trouble de la spermatogenèse. Par contre le fait d'avoir une obstruction des voies séminales n'est pas associé à une diminution significative $\mathrm{du}$ risque d'avoir un trouble de la spermatogenèse $(O R=0,835[0,583-1,196] ; p=0,3244)$.

Plusieurs facteurs de risques peuvent être présents chez un même patient. La fréquence de l'association des facteurs de risque de trouble de la spermatogenèse chez un même patient est indiquée dans le tableau 5. La fréquence des troubles de la spermatogenèse est de 64,9 $\%$ chez les patients présentant un seul facteur de risque, $68,8 \%$ chez les patients présentant plusieurs facteurs de risque $(\mathrm{p}=0,2792)$, alors qu'elle est de 38,2 \% chez les patients ne présentant aucun des facteurs de risque $(\mathrm{p}<0,0001)$.

\section{DISCUSSION}

Plusieurs études donnent des résultats concordants en ce qui concerne l'incidence de l'hypofécondité des couples. Il apparaît que $14 \%$ des couples ont des difficultés pour concevoir. L'estimation de la contribution masculine dans l'infertilité des couples se heurte à la difficulté du diagnostic de l'infertilité masculine, les données spermiologiques ne permettant pas d'établir formellement le diagnostic d'infertilité masculine dans tous les cas (revue dans [6]). Néanmoins, les données établies en France [10] ou dans le monde [2] permettent d'estimer que l'infertilité des couples est en rapport avec un facteur masculin dans plus de la moitié des cas, soit de manière isolée, soit de manière associée à un facteur féminin. Les causes d'in- fertilité masculine sont multiples et l'estimation de l'incidence des diverses causes est difficile. La comparaison des données de ces différentes études montre des variations importantes indiquées dans le tableau 6 tiré de [7]. Plusieurs facteurs peuvent expliquer ces variations. Le mode de recrutement des patients diffêre selon les études, il s'agit soit de séries de patients présentant une infertilité masculine $[1,4,5,7,8]$ ou de séries de couples infertiles dont l'homme présente des caractéristiques spermiologiques le rendant suspect de participer à l'infertilité du couple. C'est le cas de l'étude multicentrique conduite par l'Organisation Mondiale de la Santé $[6,9,11]$. Par ailleurs, il est probable que certaines données rendent compte d'un recrutement spécifique à une équipe ayant une compétence particulière pour l'une des causes d'infertilité masculine. Le chiffre élevé de varicocèle dans l'étude de Dubin et al. [4] peut être lié au fait que l'équipe travaillait sur le traitement chirurgical des varicocèles. Le chiffre élevé des pathologies endocriniennes rapportées par Nieschlag and Behre [8] peut être en relation avec l'intérêt de l'équipe pour les hypogonadismes hypogonadotropes.

Un élément semblant encore plus largement en cause dans les variations apparentes d'incidence des diverses causes d'infertilité masculine est le choix des catégories étiologiques retenues pas les auteurs et les critères utilisés pour assigner tel patient à telle catégorie étiologique. Selon les séries, un même patient sera classé dans des catégories différentes. On note que certaines catégories étiologiques ne sont pas représentées en tant que telle dans certaines séries. C'est par exemple le cas de publications $[4,5,1]$ qui ne considèrent pas de catégorie "infertilité d'origine infectieuse". Même s'il est vrai que la manière de retenir le diagnostic d'infertilité d'origine infectieuse ne fait pas l'unanimité entre les différents auteurs, les patients relevant de ce mécanisme $(55,8 \%$ de nos cas) seront alors inclus dans d'autres catégories étiologiques.

Par ailleurs, la plupart des séries publiées ne retiennent qu'une seule cause d'infertilité par patient. Dans notre expérience, la conjonction de plusieurs facteurs d'infertilité est fréquente. 
Tableau 5. Association des facteurs de risque de trouble de la spermatogenèse entre eux. Pour chaque facteur de risque, a nombre (n) et pourcentage (\% de la ligne), de cas pour lesquels le facteur de risque est isolé ou associé à un autre facteur de risque. $b$ idem pour les patients présentant le facteur de risque et un trouble de la spermatogenèse.c pourcentage des patients présentant un trouble de la spermatogenèse (\% de la colonne). $d$ Test exact de Fisher du pourcentage de troubles de la spermatogenèse selon que le facteur de risque est isolé ou associé. $A$ : étude de chaque facteur de risque individuellement. $B$ : Répartition des patients présentant un ou plusieurs facteurs de risque ou n'en présentant aucun. e Test exact de Fisher du pourcentage de troubles de la spermatogenèse selon que le patient présente un ou plusieurs facteurs de risque.

A

Facteurs étiologiques

Varicocèlea

avec trouble spermatogenèse $\mathrm{b}$

$\%$ avec trouble spermatogenèsec

Trouble descente testiculaire avec trouble spermatogenèse \% avec trouble spermatogenèse

Hyperthermie testiculaire avec trouble spermatogenèse \% avec trouble spermatogenèse

Exposition à des toxiques avec trouble spermatogenèse \% avec trouble spermatogenèse

Traumatisme testiculaire avec trouble spermatogenèse \% avec trouble spermatogenèse

Torsion testiculaire avec trouble spermatogenèse $\%$ avec trouble spermatogenèse

Orchite

avec trouble spermatogenèse

\% avec trouble spermatogenèse

Chimio-Radiothérapie avec trouble spermatogenèse $\%$ avec trouble spermatogenèse

\section{Hypospadias}

avec trouble spermatogenèse

\% avec trouble spermatogenèse

\begin{tabular}{cc}
\multicolumn{2}{c}{ Isolés } \\
$\mathbf{n}$ & $\%$ \\
306 & $71,8 \%$ \\
225 & $71,7 \%$ \\
$73,5 \%$ &
\end{tabular}

$\begin{array}{cr}266 & 63,6 \% \\ 182 & 62,5 \% \\ 68,4 \% & \end{array}$
$80 \quad 46,2 \%$
$30 \quad 36,1 \%$
$37,5 \%$

$68 \quad 48,6 \%$

$30 \quad 38,0 \%$

$44,1 \%$
$44 \quad 50,0 \%$
$20 \quad 43,5 \%$
$45,5 \%$
$10 \quad 33,3 \%$
$7 \quad 35,0 \%$
$70,0 \%$
$17 \quad 60,7 \%$
$16 \quad 61,5 \%$
$94,1 \%$
$19 \quad 73,1 \%$
$1768,8 \%$ $89,5 \%$

\begin{tabular}{cc}
\multicolumn{3}{c}{ Associés } \\
$\mathbf{n} \quad \%$ \\
$120 \quad 28,2 \%$ \\
89 & $28,3 \%$ \\
$74,2 \%$ &
\end{tabular}

$152 \quad 36,4 \%$
$109 \quad 37,5 \%$
$71,7 \%$
$93 \quad 53,8 \%$
$53 \quad 63,9 \%$
$57,0 \%$

$$
72 \quad 51,4 \%
$$

$49 \quad 62,0 \%$

$68,1 \%$

$4450,0 \%$

$26 \quad 56,5 \%$

$59,1 \%$

$20 \quad 66,7 \%$

$1365,0 \%$

$65,0 \%$

$1139,3 \%$

$10 \quad 38,5 \%$

$90,9 \%$

$\begin{array}{rr}7 & 26,9 \% \\ 7 & 29,2 \% \\ 100,0 \%\end{array}$

$6 \quad 54,5 \%$

$5 \quad 71,4 \%$

$83,3 \%$

\begin{tabular}{crr}
\multicolumn{2}{c}{ Total } & \\
$\mathbf{n}$ & $\%$ & \\
426 & $100 \%$ & \\
314 & $100 \%$ & \\
$73,7 \%$ & $>0,9999$
\end{tabular}

$418 \quad 100 \%$
$291 \quad 100 \%$
$69,6 \%$

0,5086

$173100 \%$

$83100 \%$

$48,0 \%$

0,0144

$140100 \%$

$79 \quad 100 \%$

$56,4 \%$

0,0062

$88 \quad 100 \%$

$46 \quad 100 \%$

$52,3 \%$

0,2859

$30 \quad 100 \%$

$20 \quad 100 \%$

$66,7 \% \quad>0,9999$

$28 \quad 100 \%$

$26 \quad 100 \%$

$92,9 \%$

$>0,9999$

$26 \quad 100 \%$

$24100 \%$

$92,3 \%$

$>0,9999$

$11100 \%$

$7 \quad 100 \%$

$63,6 \%$

0,2424

\title{
B
}

Facteurs de risques : aucun

$\begin{array}{lcc}\text { Ensemble de patients } & \text { n } & \%\end{array}$

Trouble spermatogenèse $\quad 388 \quad 35,8 \%$

\% trouble spermatogenèse $38,2 \%$
$2 \quad 28,6 \%$

$40,0 \%$

\begin{abstract}
-
\end{abstract}


C'est en particulier le cas en ce qui concerne l'association de mécanismes semblant à priori indépendants comme les troubles de la spermatogenèse et la pathologie post-testiculaire (Figure 1), au sein de laquelle la pathologie infectieuse est particulièrement fréquente. La présence d'une pathologie infectieuse et la présence d'un facteur de risque de trouble de la spermatogenèse apparaissent deux caractéristiques indépendantes chez nos patients ( $\mathrm{p}=0,1703$; Tableau 7)

Dans chacune des séries publiées on retrouve une catégorie, souvent numériquement importante, qualifiée d'idiopathique". Il est à noter que, selon le terme auquel s'applique l'adjectif "idiopathique", la population décrite est différente. L'infertilité "idiopathique" correspond à une infertilité de couple d'origine inconnue, c'est-à-dire une infertilité "inexpliquée", ce qui implique que les examens complémentaires, dont le spermogramme, sont normaux. L'oligoasthénospermie "idiopathique" correspond cette fois-ci à une altération du spermogramme dont l'origine est inconnue, ce qui laisse sous-entendre que l'on ne dispose pas d'argument en faveur, en particulier, d'un trouble de la spermatogenèse, sinon on parlerait d'oligoasthénospermie par trouble de la spermatogenèse. Enfin il peut s'agir d'un trouble de la spermatogenèse "idiopathique". Dans ce cas, on dispose d'arguments en faveur d'un trouble de la spermatogenèse, mais son origine reste mystérieuse, le caryotype est normal, il n'y a pas de microdélétion du chromosome Y ni de facteur étiologique (varicocèle, cryptorchidie etc).

Il apparaît utile de considérer plusieurs niveaux de classification. Bien qu'imparfaite sur le plan pronostique et peu spécifique sur le plan étiologique, la classification spermiologique est unanimement utilisée. Une classification physiopathologique comme celle citée récemment par de Kretzer et Baker [3], dont nous nous sommes inspirés ici, permet de considérer le mécanisme de l'altération du spermogramme, même si la cause en reste encore imprécise à l'échelon moléculaire. La classification étiologique s'affine au fur et à mesure de la découverte de nouveaux mécanismes d'altération de la fonction de reproduction masculine.
Dans notre série, un trouble de la spermatogenèse est identifié, sur des critères indépendants des facteurs de risques, chez $52 \%$ des hommes consultant pour infertilité masculine. Si cette proportion est appliquée aux 8\% (14\% x 59\%) des hommes vivant en couple et qui présentent un facteur masculin d'infertilité [10], on conclut que $4 \%$ des hommes vivant en couple présentent un trouble de la spermatogenèse.

Le trouble de la spermatogenèse est isolé dans $43 \%$ des cas (soit $23 \%$ des patients vus en consultation d'infertilité masculine) et associé à des altérations "post-testiculaires" (infectieuses/inflammatoires, autoimmunes et/ou obstructives) dans 57\% des cas (soit 30\% des patients).

Un facteur étiologique est trouvé pour $64 \%$ des patients présentant un trouble de la spermatogenèse. Plusieurs facteurs étiologiques de troubles de la spermatogenèse s'associent chez un même patient dans $15 \%$ des cas de troubles de la spermatogenèse.

À l'opposé, pour $36 \%$ des patients, le trouble de la spermatogenèse n'est pas associé à un facteur de risque cliniquement identifiable. Après les causes génétiques (cytogénétiques et microdélétions du chromosome $\mathrm{Y}$; Tableau 8), les facteurs de risque les plus fortement associés aux troubles de la spermatogenèse sont : d'une part les antécédents d'orchite ourlienne et de radiothérapies-chimiothérapies, situations relativement peu fréquentes mais génératrices de troubles de la spermatogenèse dans une proportion élevée de cas ( $>90 \%$ ) et d'autre part les varicocèles et les troubles de la descente testiculaire, qui sont les facteurs de risques les plus fréquents, mais sont moins régulièrement associés à un trouble de la spermatogenèse.

Cette étude indique que l'infertilité masculine procède de l'association de plusieurs facteurs étiologiques, agissant par des mécanismes indépendants, pour un nombre important de patients. C'est en particulier le cas pour l'association de troubles de la spermatogenèse et d'altérations post-testiculaires, en particulier (post-)infectieuses. Elle attire en outre l'attention sur l'intérêt d'une analyse des mécanismes physiopathologiques pour établir les relations de cause à effet entre les facteurs étiologiques et l'infertilité. 
Tableau 6 : Pourcentages de diverses causes d'infertilité masculine : valeurs extrêmes relevées dans une compilation de 7 séries publiées [7]. \%min:\% minimal donné dans une des 7 études; \%Max : \% maximal donné dans une des 7 études ; "\%moyen": moyenne des pourcentages, pondéré selon le nombre de patients dans les différentes études; $n$ : nombre d'études considérant cette catégorie étiologique.

Causes endocrines

Varicocèle

Déficit testiculaire

Cryptorchidie

Cancer du testicule

Causes infectieuses

Auto-immunisation

Azoospermie obstructive

Dysfonctions sexuelles

Causes systémiques

Causes diverses

Idiopathiques

\section{[\%min-\%Max]}

[ $0,6-9,7]$

[15,4-40,3 ]

[ $4,0-14,0$ ]

[ $4,4-8,0]$

[ $1,4-2,1]$

[ $8,5-13,7]$

[ $0,8-6,2$ ]

[ $1,5-7,4$ ]

$[0,5-6,7]$

[ $1,4-5,2]$

[ $0,7-19,6]$

$[\underline{5,4-51,5}]$

$\begin{array}{cc}\text { “moyen" } & \mathbf{n} \\ 5 & 7 \\ 24 & 7 \\ 10 & \underline{4} \\ 7 & 5 \\ 2 & \underline{2} \\ 11 & \underline{4} \\ 5 & 6 \\ 3 & 6 \\ 5 & 6 \\ 4 & 3 \\ 9 & 6 \\ 36 & 7\end{array}$

Tableau 7 : Relation entre les facteurs de risques de trouble de la spermatogenèse et la pathologie infectieuse/inflammatoire : $n$ : nombre de patients; \% pourcentage de patients rapporté au nombre total de patients (2072). On ne met pas en évidence de liaison significative entre les facteurs de risque de troubles de la spermatogenèse et l'infection (test exact de Fisher : p=0,1703).

\begin{tabular}{lccccccc} 
& \multicolumn{2}{c}{$\begin{array}{c}\text { Pas de f. de risque } \\
\text { de tr. spermatog. }\end{array}$} & \multicolumn{2}{c}{ F. de risque } \\
& de tr. spermatog. & \multicolumn{2}{c}{ Total } \\
& $\mathbf{n}$ & $\%$ & $\mathbf{n}$ & $\%$ & n & $\%$ \\
Infection/inflam. & 583 & $28,1 \%$ & 574 & $27,7 \%$ & 1157 & $55,8 \%$ \\
Pas d'infect/inflam. & 433 & $20,9 \%$ & 482 & $23,3 \%$ & 915 & $44,2 \%$ \\
Total & 1016 & $49,0 \%$ & 1056 & $51,0 \%$ & 2072 & $100 \%$
\end{tabular}

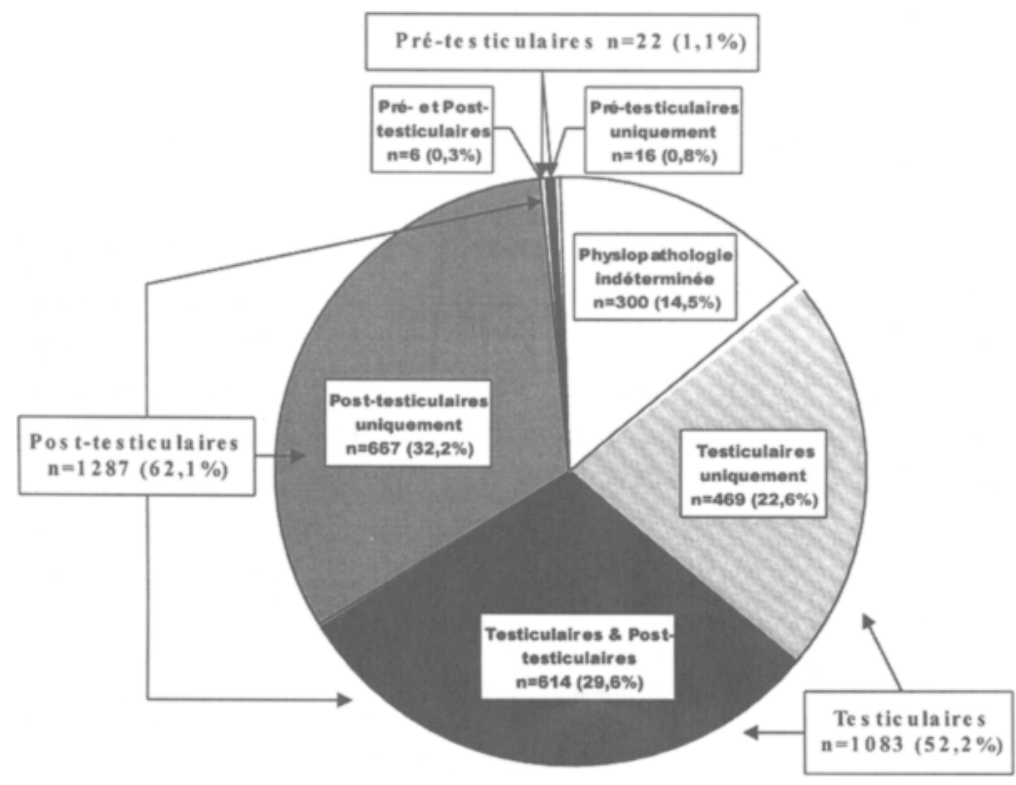

Figure 1. Associations des catégories physiopathologiques. 
Tableau 8. Troubles de la spermatogenèse d'origine génétique. Un caryotype sanguin a été réalisé pour 820 patients présentant une azoospermie ou entrant dans un programme de microinjection. La recherche de microdélétion du bras long du chromosome $Y$ a été réalisée pour 268 patients ayant une concentration de spermatozoüdes $<5.106 / \mathrm{ml}$. a pourcentage de patients présentant l'anomalie génétique rapporté au nombre de patients étudiés ; b pourcentage de patients présentant un trouble de la spermatogenèse parmi les patients présentant l'anomalie génétique ; $c$ pourcentage de patients présentant l'anomalie génétique parmi les patients présentant un trouble de la spermatogenèse ( $n=546$ pour les causes cytogénétiques; $n=231$ pour les microdélétions) ; d 6 patients présentant une anomalie caryotypique autosomique n'ont pas de trouble de la spermatogenèse identifiable.

\section{$\% 1$ \\ patientsa \\ $\%$ de troubles spermatogenèseb \\ Trble Sptgc}

$88 \% \mathrm{~d}$ $8,1 \%$

Caryotype anormal $(n=50)$

$6,1 \%$

$2,3 \%$

$0,7 \%$

$3,0 \%$
Klinefelter $(\mathrm{n}=19)$

Autres $(n=6)$

Autosomiques $(n=25)$
Causes cytogénétiques ( 820 caryotypes)

Gonosomiques

Microdélétions du chromosome $\mathbf{Y}$ (268 recherches de microdélétions)

Yq microdélétions $(n=11)$

$4,1 \%$

$100 \%$

$4,8 \%$

Yq microdelétions $(\mathrm{n}=11)$

(1)

\section{Remerciements}

Ce travail a été soutenu par le réseau de recherche clinique INSERM $n^{\circ} 493010$ et la commission recherche des Hospices Civils de Lyon.

\section{RÉFÉRENCES}

1. BAKER H.W.G., BURGER H.G., DE KRETSER D.M., HUDSON B. : Relative incidence of etiological disorders in male infertility. In : Santen R.J., Swerdloff R.S. eds. Male Reproductive Dysfunction: Diagnosis Management of Hypogonadism, Infertility and Impotence. New York, Dekker, 1986 : 341-372.

2. CATES W., FARLEY T.M.M., ROWE P.J. : Worldwide patterns of infertility: is Africa different ? Lancet, 1985, ii : 596-598.

3. DE KRETSER D.M., BAKER H.W.G. : Infertility in men: Recent advances and continuing controversies. J. Clin. Endocrinol. Metab., 1999, 84 : 3443-3450.

4. DUBIN L., AMELAR R.D. : Etiologic factors in 1,294 consecutive cases of male infertility. Fertil. Steril., 1971, $22:$ 469-474.

5. GREENBERG S.H., LIPSHULZ L.I., WEIN A.J. :
Experience with 425 subfertile male patients. J. Urol., 1978, 119 : 507-510.

6. IRVINE D.S. : Epidemiology and aetiology of male infertility. Hum. Reprod., 1998, 13 (suppl.1) : 33-44.

7. MARTIN DU PAN R.C., BISCHOF P., CAMPANA A., MORABIA A. : Relationship between etiological factors and total motile sperm count in 350 infertile patients. Arch. Androl., 1997, 39 : 197-210.

8. NIESCHLAG E., BEHRE H.M. : Male infertility due to testicular dysfunction. In : Drife J.O., Templeton A.A. eds. Infertility Proceedings of the 25th Study Group of the Royal College Obstetricians and Gynaecologists. London, Springer, 1992 : 65-79.

9. THE ESHRE CAPRI WORKSHOP GROUP : Male sterility and subfertility: guidelines for management. Hum. Reprod., 1994, 9 : 1260-1264.

10. THONNEAU P., MARCHAND S., TALLEC A., et al. : Incidence and main causes of infertility in a resident population (1.850.000) of three French regions (19881989). Hum. Reprod., 1991, $6: 811-816$.

11. WORLD HEALTH ORGANIZATION : Towards more objectivity in diagnosis and management of male infertility. Int. J. Androl., 1987, 7 (suppl) : 1-53. 


\begin{abstract}
Involvement of spermatogenic deficiency in male infertility.

H. LEJEUNE, M. BENCHAIB, V. BIED, A. BOUCHER, D. BOULIEU, J.C. CZYBA, P. GALLOTLAVALLEE, J.F. GUERIN, S. HADJ, J. LORNAGE, I. PLOTTON, M. PUGEAT, B. SALLE.
\end{abstract}

According to Thonneau et al., (1991), 14\% of couples are subfertile. In at least $59 \%$ of the cases, a male factor was involved. This indicates that $8 \%(14 \% \times 59 \%)$ of men are hypofertile. Since several causes can induce male infertility and because of the heterogeneous criteria and classifications used in the literature, the percentage of each etiologic factor has not been very precisely established. In a population of 2072 consecutive patients we identified an alteration of spermatogenesis in $52 \%$ of the cases. This indicates that about $4 \%$ of men might have a spermatogenic problem. The spermatogenic insufficiency was isolated in $43 \%$ of the cases (i.e. $23 \%$ of the total population) or associated with post-testicular causes of male infertility (infectious/inflammatory; autoimmune; obstructive) in $57 \%$ of the cases (i.e. $30 \%$ of the total population studied). An etiologic factor is clinically identified for $64 \%$ of the patients presenting with a spermatogenic insufficiency. The most relevant risk factors linked to spermatogenic alteration were history of mumps orchitis (OR $[\mathrm{IC95} \%]=14,6[3,4-62,3])$, history of radiotherapy-chemotherapy $(\mathrm{OR}=14,7 \quad[3,4-63,2])$. These situations were found with a low frequency $(1,4 \%$ and $1,3 \%$ of the cases respectively) but provoked a spermatogenic alteration in a large majority of cases $(92,9 \%$ and $92,3 \%$ respectively). On the other hand, varicoceles $(\mathrm{OR}=3,7[2,9-4,8])$ and troubles in testicular descend $(\mathrm{OR}=2,9[2,3-3,7])$, were more frequent $(20,6 \%$ and $20,1 \%$ of the cases respectively), but less frequently associated with spermatogenic insufficiency (in 73,7 and $69,6 \%$ of the cases).

Key-words: male infertility - spermatogenesis physiopathology - etiology 